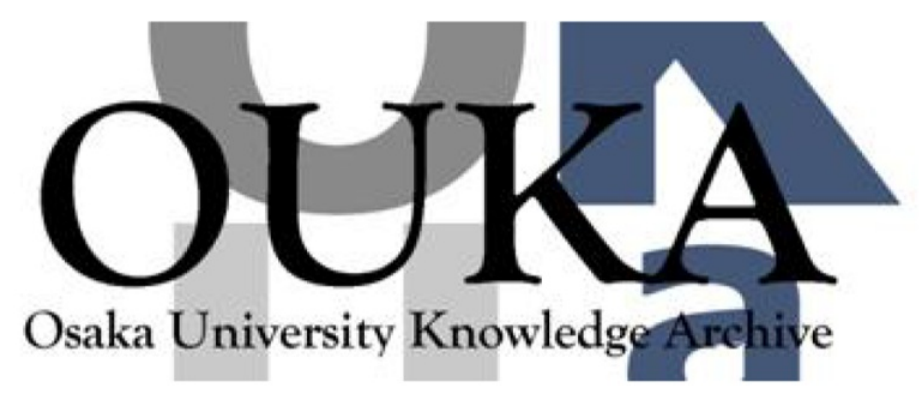

\begin{tabular}{|c|l|}
\hline Title & $\begin{array}{l}\text { Stacking and translation of microscopic } \\
\text { particles by means of } 2 \times 2 \text { beams emitted from a } \\
\text { vertical-cavity surface-emitting laser array }\end{array}$ \\
\hline Author(s) & Sumiyama, Fumika; Ogura, Yusuke; Tanida, Jun \\
\hline Citation & Applied physics letters. 82(18) p. 2969-p. 2971 \\
\hline Issue Date & $2003-05-05$ \\
\hline oaire:version & VoR \\
\hline URL & https://hdl. handle.net/11094/3037 \\
\hline rights & \\
\hline Note & \\
\hline
\end{tabular}

Osaka University Knowledge Archive : OUKA

https://ir. Library. osaka-u. ac. jp/

Osaka University 


\section{$1 D$ Applied Physics \\ Letters}

\section{Stacking and translation of microscopic particles by means of $2 \times 2$ beams emitted from a vertical-cavity surface-emitting laser array}

Fumika Sumiyama, Yusuke Ogura, and Jun Tanida

Citation: Appl. Phys. Lett. 82, 2969 (2003); doi: 10.1063/1.1570939

View online: http://dx.doi.org/10.1063/1.1570939

View Table of Contents: http://apl.aip.org/resource/1/APPLAB/v82/i18

Published by the American Institute of Physics.

\section{Related Articles}

Effect of shell thickness on two-photon absorption and refraction of colloidal CdSe/CdS core/shell nanocrystals Appl. Phys. Lett. 99, 231903 (2011)

Local structure and magnetic properties of Mn-doped 3C-SiC nanoparticles

Appl. Phys. Lett. 99, 222512 (2011)

Reorganization of Au nanoparticle Langmuir-Blodgett films on wet chemically passivated $\mathrm{Si}(001)$ surfaces J. Appl. Phys. 110, 114302 (2011)

Growth of [010] oriented - $\mathrm{MoO} 3$ nanorods by pulsed electron beam deposition

Appl. Phys. Lett. 99, 223104 (2011)

Gain assisted nanocomposite multilayers with near zero permittivity modulus at visible frequencies Appl. Phys. Lett. 99, 221107 (2011)

\section{Additional information on Appl. Phys. Lett.}

Journal Homepage: http://apl.aip.org/

Journal Information: http://apl.aip.org/about/about_the_journal

Top downloads: http://apl.aip.org/features/most_downloaded

Information for Authors: http://apl.aip.org/authors

\section{ADVERTISEMENT}

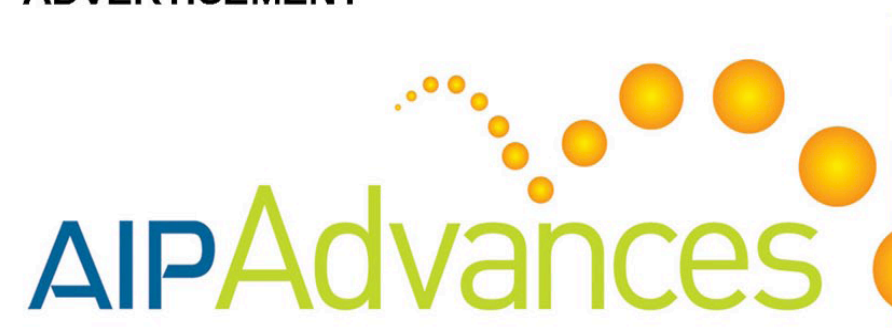

Submit Now

\section{Explore AIP's new \\ open-access journal}

Article-level metrics now available

Join the conversation!

Rate \& comment on articles 


\title{
Stacking and translation of microscopic particles by means of $2 \times 2$ beams emitted from a vertical-cavity surface-emitting laser array
}

\author{
Fumika Sumiyama, ${ }^{\text {a) }}$ Yusuke Ogura, and Jun Tanida \\ Department of Information and Physical Sciences, Graduate School of Information Science and Technology, \\ Osaka University, 2-1 Yamadaoka, Suita, Osaka 565-0871, Japan
}

(Received 9 December 2002; accepted 4 March 2003)

\begin{abstract}
We report on an optical manipulation method to achieve stacking of multiple particles by simultaneous emission of vertical-cavity surface-emitting laser sources. In the experiment, up to seven polystyrene particles of $10-\mu \mathrm{m}$ diameter were stacked vertically by illuminating $2 \times 2$ beams. Furthermore, we could translate the particles keeping the stacked state without mechanical movement. The maximum number of stackable particles was obtained when the focal spots of the beams were located $20 \mu \mathrm{m}$ above the sample stage. We also found that the relation between the spot pitch and the particle diameter is an important factor to achieve stacking. This manipulation method is expected to be useful for microfabrication of a three-dimensional structure and collective translation of materials capsuled in microparticles. (c) 2003 American Institute of Physics.
\end{abstract}

[DOI: $10.1063 / 1.1570939]$

An optical manipulation method, which uses radiation pressure induced by interaction between light and an object, is a useful tool due to the capability of translating small objects without mechanical contact or distraction. ${ }^{1}$ This technique has been used in a wide range of applications, such as real-time observation of a single rotating biomolecule, ${ }^{2}$ measurement of the tensile force with knotting a DNA filament, ${ }^{3}$ and investigation of the mechanism of molecular motor motion. ${ }^{4}$ To manipulate multiple objects, generation of multiple light spots at a plane is straightforward, and it provides us an assembling method of microparticles in two dimensions. Computer-generated holograms (CGHs) are often used for the purpose, and an array of $10 \times 10$ particles, for example, was constructed. ${ }^{5}$ The generalized phase-contrast method, by which the intensity pattern for trapping corresponds to an input phase filter directly, was also applied to arrange the particles. ${ }^{6}$

Techniques for fabricating structures composed of microscopic objects are very important in contemporary science. For example, various interesting properties of photonic crystals can be embodied by fabrication of structures designed in multiple dimensions. ${ }^{7}$ On a photonic crystal, specific arrangement of materials is crucial and precise fabrication holds a key for practical applications. In this case, although a structure with one or two dimensions also shows interesting functionalities, three-dimensional (3D) structures become important to obtain strong nonlinearity, such as perfect light confinement.

Recently, it was reported that 3D microscopic structures were constructed with adequately controlled radiation pressure based on different methods. Curtis et al. have advanced the method using $\mathrm{CGH}$ to arrange the individual particles at different axial planes. ${ }^{8}$ Use of a high-order mode of laser beam is another solution to generate the light distribution to construct the 3D structure. For example, nine particles were stacked in the vertical direction by use of zeroth-order Bessel

${ }^{a)}$ Electronic address: sumiyama@ist.osaka-u.ac.jp light beams, ${ }^{9}$ and a 3D structure was created by intensity distribution of four beams that were generated by interference of two Lagguere-Gaussian light beams. ${ }^{10}$ Although these methods are interesting and sophisticated, specially designed optical elements, such as a holographic optical element, must be prepared for the specific optical distribution.

In this letter, we propose a method for fabricating a 3D structure by means of vertical-cavity surface-emitting laser (VCSEL) array optical manipulation. ${ }^{11,12}$ We report that we can stack and translate a multiple number of microscopic objects with illumination of $2 \times 2$ beams emitted from a VCSEL array. Due to simplicity of the controlling method, this technique is expected to be useful to make the hardware for 3D fabrication simple and compact.

Figure 1 illustrates the experimental setup of the VCSEL array manipulation system. We use a VCSEL array device

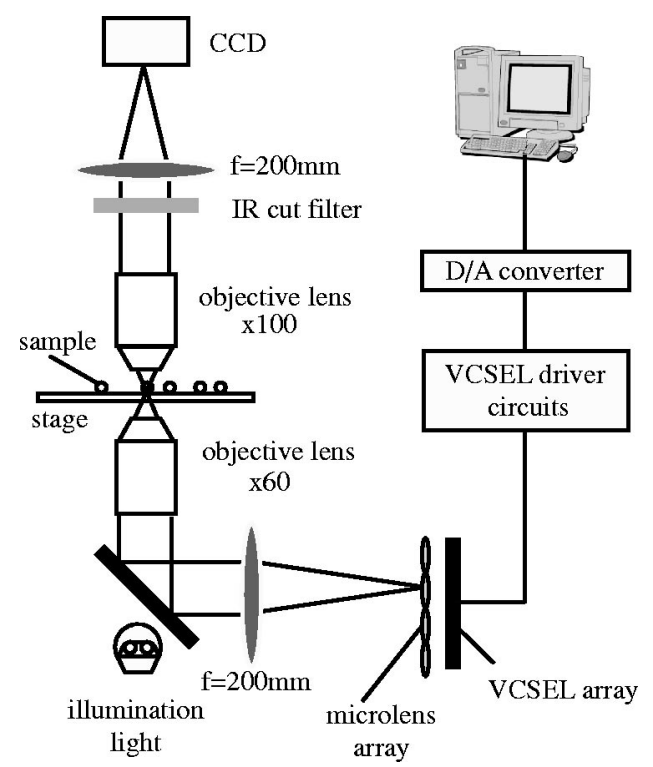

FIG. 1. Experimental setup of VCSEL array trapping. D/A: digital-toanalog. 


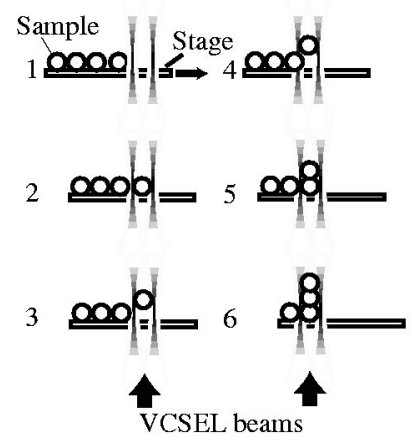

(a)

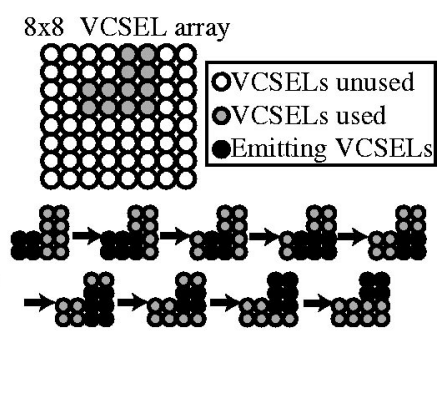

(b)
FIG. 2. Experimental method of stacking and translation for microparticles. (a) A sequence of stacking and (b) an emission sequence of the VCSELs for the nonmechanical translation.

(an $854 \pm 5 \mathrm{~nm}$ wavelength, $8 \times 8$ pixels, $250-\mu \mathrm{m}$ pixel pitch, $15-\mu \mathrm{m} \phi$ aperture, and $5.1 \mathrm{~mW} \max$ emission power) provided from NTT Photonics Laboratory. Emission distribution of the VCSEL array is controlled with a personal computer through a digital-to-analog converter and VCSEL driver circuits. Each of the VCSEL array was driven by $500-\mathrm{Hz}$ rectangular wave signal to prevent overload of the device. We set a microlens array in front of the VCSEL to reduce loss of the beam intensity. The focal length and the lens pitch of the microlens array are 720 and $250 \mu \mathrm{m}$, respectively.

We used a water-immersible, long-distance objective lens (Olympus LUMPlan, 60×, $0.90 \mathrm{NA}$ ) as the focusing lens. The pixels of the VCSEL array were imaged onto the sample plane with magnification of $1 / 67$. Thus, the optical spot pitch on the sample plane is $3.75 \mu \mathrm{m}$, the average intensity of light is $1.0 \mathrm{~mW}$, and the diameter of the spot is 2.6 $\mu \mathrm{m}$. The sample objects were polystyrene particles (Polysciences, Inc., polybead polystyrene microspheres) with 1.60 refractive index, and were dispersed in water.

Figure 2(a) shows a sequence of a experiment for stacking microparticles. First, we turned on $2 \times 2$ pixels of the VCSEL array and focused the beams around a microparticle. The microparticle was then lifted by the light pressure. Next, we moved the sample stage to focus the beams around another microparticle. The microparticle moved under the microparticle previously captured, and these particles lined up vertically. Repeating this process, we could stack microparticles along with a vertical space surrounded by $2 \times 2$ beams.

At the next stage, we attempted to translate the stacked particles by changing the emitting pixels of the VCSEL array. The VCSEL pixels were turned on from the lower left to the upper right as shown in Fig. 2(b). To prevent break down of the stacked structure, 4 or 6 pixels were turned on at a time. By the method, we achieved translation of the particles while maintaining the stacked structure without mechanical movement. Figure 3 shows the experimental result of stacking of seven microparticles (10 $\mu \mathrm{m}$ in diameter) with focusing the beams at a plane $20-\mu \mathrm{m}$ distant from the sample plane: (a) is the initial status ( $\mathrm{X}$ is the origin of the stage), (b) shows that the seven microparticles are stacked at the position marked $\cdot$, and (c) shows that the stacked particles are translated without mechanical movement. After turning off the VCSEL sources, the stacked structure was broken down

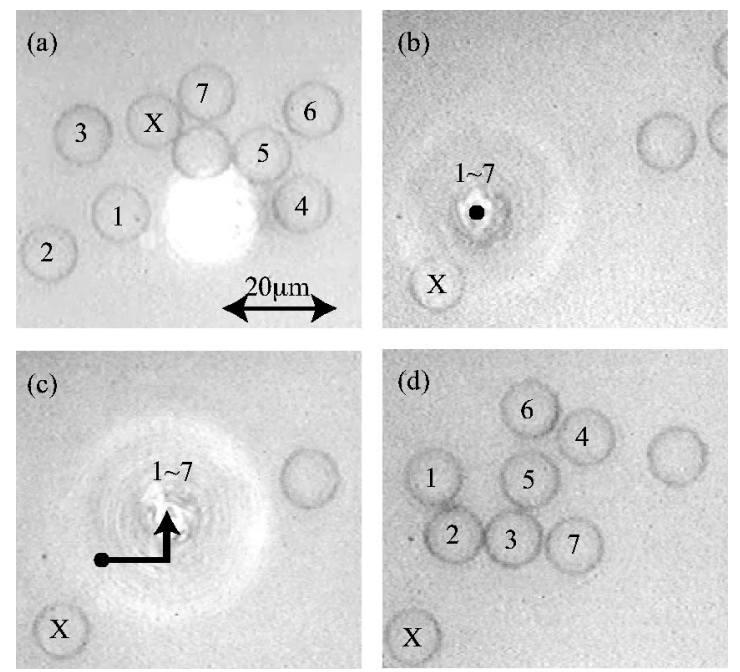

FIG. 3. Experimental result of stacking particles. $\mathrm{X}$ is the origin of the stage. (a) Initial status, (b) seven microparticles were stacked at the position of mark $\cdot$, (c) the particles were translated along the arrow, and (d) the particles were released after turning off the VCSEL sources.

and the microparticles were spread on the sample stage [Fig. $3(d)]$.

We performed the experiment several times with the same conditions and we could then stack, without mechanical translation up to seven microparticles. These results imply that the microparticles follow the movement of the beams emitted from the VCSEL array and that the stacked particles are translated simultaneously.

To investigate the effect of the particle size, three kinds of particles with 3-, 6-, and 10- $\mu \mathrm{m}$ diameters were used in the stacking experiment. Among the different sizes of particles, we could stack the most number of particles when we used $10-\mu \mathrm{m}$-diameter particles. The reason is thought to be as follows. The distribution of the $2 \times 2$ VCSEL beams on the sample plane (spot pitch $3.75 \mu \mathrm{m}$ ) is small enough to be covered by the particle of $10-\mu \mathrm{m}$ diameter. The particle receives light pressure equally from all the beams. Therefore, the particles were stabilized around the center of the four spots by the horizontal light pressure although levitated.

The maximum number of the stackable particles were three for $6-\mu \mathrm{m}$-diameter particles and one (not stacked) for 3 - $\mu$ m-diameter particles. In this case, we could not achieve translation of the particles while maintaining the structure. The particle was trapped by the nearest beam because the size of these microparticles is smaller than the distribution of the $2 \times 2$ beams. Therefore, each microparticle was trapped at a different position by the four beams.

To overcome the problem, we exchanged the focusing lens into a $100 \times$ objective lens. By using this lens, the spot pitch on the sample stage was decreased from $3.75 \mu \mathrm{m}$ to $2.25 \mu \mathrm{m}$. We attempted again to stack and translate $6-\mu \mathrm{m}-$ diameter particles, and we could stack four 6 - $\mu$ m-diameter particles and translate keeping the stacked structure. This result shows that the condition to create a stacking structure depends on relationship between the size of particles and the pitch of beams.

To find a relationship between the position of the focal point of beams and the maximum number of microparticles that could be stacked, we changed the focal position distant 


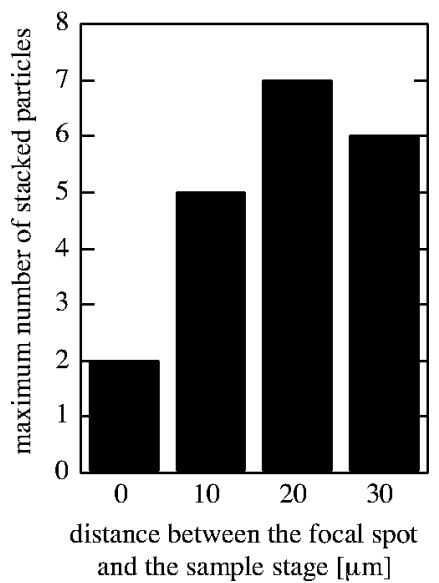

FIG. 4. Relationship between the position of the focal point of beams and the maximum number of microparticles.

from the sample stage. With different distances $Z$ between the focal and the sample stage, we counted the number of stackable particles. The result is shown in Fig. 4. The result indicates that the number of stackable particles depends on $Z$ and that the maximum number is obtained by the defocus condition in which the focus is located above the stage.

Changing the position of the focal point modifies the optical field affecting to the bottom particle. To create a desired structure, we must control illuminating pattern of the VCSEL beams. In addition, the lens effect of a particle must be considered in the beam propagation. Due to the lens effect of each particle, the propagating beam is expected to take complicated paths. Therefore, constructing an appropriate model and analyzing the effective force onto the particles are important issues for practical application of the proposed method.

The most important achievement in this report is the demonstration of stacking microparticles with illumination of $2 \times 2$ beams emitted from a VCSEL array. The emission distribution of the VCSEL array is easily changed by an electrical control, so that we can achieve stacking of microparticles and translation of the stacked particles, while keeping the structure, without mechanical movement. Using $2 \times 2$ beams illuminated on microparticles, we could line up the particles vertically and translate them in the horizontal direction. Furthermore, it was confirmed that setting the focal point apart from the sample stage is an effective method to increase the number of stacked particles. Although the proposed method is a modification of our previous work, ${ }^{11,12}$ it provides potentially useful applications. For example, it is expected to contribute to development of compact and simple microfabrication systems. We can fabricate 3D structures nonmechanically with arrangement of the stacked particles. Also, collective translation of materials is achieved by capsuling them in microparticles.

The VCSEL array was supplied by NTT Photonics Laboratory through the System Photo-Electronics Consortium in Japan. The authors appreciate the support of the consortium for their studies on optoelectronic application systems.

${ }^{1}$ A. Ashkin, J. M. Dziedzic, J. E. Bjorkholm, and S. Chu, Opt. Lett. 11, 288 (1986).

${ }^{2}$ Y. Harada, O. Ohara, A. Takatsuki, H. Itoh, N. Shimamoto, and K. Kinosita Jr, Nature (London) 409, 113 (2001).

${ }^{3}$ Y. Arai, R. Yasuda, K. Akashi, Y. Harada, H. Miyata, K. Kinosita, Jr., and H. Itoh, Nature (London) 399, 446 (1999).

${ }^{4}$ K. Kitamura, M. Tokunaga, A. H. Iwane, and T. Yanagida, Nature (London) 397, 129 (1999).

${ }^{5}$ P. Korda, G. C. Spalding, E. R. Dufresne, and D. G. Grier, Rev. Sci. Instrum. 73, 1956 (2002).

${ }^{6}$ R. L. Eriksen, P. C. Mogensen, and J. Gluckstad, Opt. Lett. 27, 267 (2002).

${ }^{7}$ S. Noda, M. Yokoyama, M. Imada, A. Chutinan, and M. Mochizuki, Science 293, 1123 (2001).

${ }^{8}$ J. E. Curtis, B. A. Koss, and D. G. Grier, Opt. Commun. 207, 169 (2002).

${ }^{9}$ J. Arlt, V. Garces-Chavez, W. Sibbett, and K. Dholakia, Opt. Commun. 197, 239 (2001)

${ }^{10}$ M. P. MacDonald, L. Paterson, K. Volke-Sepulveda, J. Arlt, W. Sibbett, and K. Dholakia, Science 296, 1101 (2002).

${ }^{11}$ Y. Ogura, K. Kagawa, and J. Tanida, Appl. Opt. 40, 5430 (2001).

${ }^{12}$ Y. Ogura, N. Shirai, and J. Tanida, Appl. Opt. 41, 5645 (2002). 